\title{
A Review on the neuroendocrine regulation of cytokine secretion: Possible modulation of the cytokine network by the pineal hormone melatonin and cannabidiol
}

\author{
Paolo Lissoni*, Franco Rovelli, Giusy Messina, AlejandraMonzon, Sonja Pensato, Roberto Trampetti, Giorgio Porro, Giorgio Maestroni, \\ Nicoletta Merli and Giuseppe Di Fede
}

Institute of Biological Medicine, Milan, Italy

\begin{abstract}
Today it is known that most human systemic diseases, including neoplastic, autoimmune and cardiovascular pathologies, would be due to an alteration of the cytokine network, which plays a fundamental role in the regulation not only of the immune system, but of the overall main biological systems. At present, it is possible to modulate and regulate the cytokine network by the exogenous administration of those cytokines, whose endogenous secretion is abnormally diminished, or in contrast by the injection of monoclonal antibodies against those cytokines, whose endogenous production is excessively high. However, since cytokine secretion is physiologically under a neuroendocrine regulation, a new clinical approach to influence the cytokine network could consist of the exogenous administration of the major neurohormones or neuroactive agents involved in the neuroendocrine control of the immune functionless. At present, the pineal indole hormone melatonin and cannabidiol would represent some of the most promising agents, also because of their complete lack of toxicity and low cost.
\end{abstract}

\section{Introduction}

Today, it is known that the human systemic diseases, including cancer and autoimmunity, are depending on several alterations in cytokine secretions, which allow a chronic inflammatory status [1]. In fact, both cancer and autoimmunity are characterized by abnormally high blood levels of the main pro-inflammatory cytokines, including IL-1 beta, IL-6, TNF-alpha and IL-17 [2,3]. Then, the main difference between cancer and autoimmune pathologies would namely regard the profile of those cytokines, which exert both anti-inflammatory effects and an immunosuppressive activity on the anticancer immunity, namely IL-10 and TGF-beta, mainly produced by the regulatory T lymphocytes ( $\mathrm{T}$ reg) [4], and in the less manner by the M2 subtype of macrophages [5], since both TGF-beta and IL-10 have been proven to be abnormally high in the metastatic neoplastic diseases by reflecting an enhanced $\mathrm{T}$ reg system activation [6], and low in the autoimmune diseases [7], and the evidence of an increase in TGF-beta and IL-10 in the autoimmunity has appeared to predict a control of disease and a better prognosis [7]. According to the present clinical approaches in the treatment of systemic human diseases, an eventual abnormal secretion of a specific cytokine may be controlled by the injection of other cytokines with opposite effects, or by the administration of monoclonal antibodies against a specific cytokine, such as TNF-alpha in the rheumatoid arthritis. On the contrary, according to the recent discoveries in the area of psychoneuro-endocrino-immunology (PNEI) demonstrating hat the immune responses are physiologically under a psycho-neuroendocrine control, it could be possible from at least a theoretical point of view to influence the immune response by influencing the cytokine network not only by directly acting on the secretion or on the activity of some specific cytokine, but also by influencing the neuroendocrine control of the cytokine network. According to the knowledgments available up to now, within the complexity of the neuroimmunology, it is possible to identify two major neuroendocrine functional systems involved in the psychoneuroendocrine regulation of the immune system, consisting of the brain opioid system-pituitary axis [8-10] and the brain cannabinergic system-pineal axis [11-13], respectively provided by an immunosuppressive and an immunostimulatory role, respectively.

\section{Immune effects of brain opioid and cannaninergic sys- tems, and pineal gland}

At present, it has been well demonstrated that the opioid system, namely the mu-agonist one, has been proven to inhibit IL-2 and IL12 secretion, and to stimulate that of IL-10 (8-10). Since IL-2 and IL12 represent the two main anticancer immunostimulating cytokines in humans [14,15], and IL-10 one of the main immunosuppressive cytokines, it is a clinical evidence that mu-opioids may suppress the anticancer resistance and promote tumor growth and dissemination. On the same way, the activation of beta-adrenergic system has appeared to inhibit IL-2 and IL-12 secretion, as well as to exert a lymphocytolitic activity $[9,10]$. Therefore, beta-adrenergic antagonists could potentially improve the immune functions. On the other hand, the pineal gland, which is connected to the brain cannabinergic system [11],has appeared to stimulate IL-2 and IL-12 secretion by melatonin (MLT) [12], that represents its most investigated indole hormone [14], while the cannabinoid agents, including the endogenous cannabinoid agonists anandamide and 2-arachydonil-glycerol [15], and the exogenous ones, namely tetra-hydro-cannabinol (THC), may inhibit

${ }^{\star}$ Correspondence to: Paolo Lissoni, Institute of Biological Medicine, Milan, Italy, E-mail: paolo.lissoni@gmx.com

Key words: cannabidiol, cannabinoids, cannabinoid system, fatty acid amide hydrolase, melatonin, neuroimmunomodulation, pineal gland

Received: May 27, 2019; Accepted: June 06, 2019; Published: June 10, 2019 
IL-17 secretion from TH-17 lymphocytes, as well that of TNF-alpha and IL-6 from macrophages [16]. With respect to the endogenous cannabinoid system, Cannabis plant may produce another biologically important molecule, the cannabidiol (CBD), which is not a cannabinoid agonist, and this activity is due to the inhibition of the fatty acid amide hydrolase (FAAH), the enzyme involved in cannabinoid degradation, with a consequent increase in brain cannabinoid content $[17,18]$. Moreover, in contrast to the mu-opioidagonists, which inhibit IL-2 and IL-12 secretions and stimulate IL-10 release [8], CBD has been proven to stimulate IL-12 and IL-2 secretions and to inhibit that of IL-10 [19], by improving the antitumor immunity, which is stimulated by IL-2 and IL-12, and inhibited by IL-10 and TGF-beta [20].

\section{The antitumor mechanisms of the pineal indole hormone melatonin}

The pineal hormone MLT, which is mainly produced during the dark phase of the day with a consequent well defined light/dark circadian rhythm [14] and whose nocturnal secretion has been proven to progressively decline with cancer progression [21] by representing the main cancer progression-related endocrine deficiency, has appeared to play a fundamental role in the maintenance of the natural immunobiological resistance against cancer onset and growth [22], since it is able to play an anticancer action through different mechanisms, including a direct cytostatic cytotoxic effect of cancer cell expressing MLT receptor (MT-R), an immunostimulatory role consisting of a direct stimulation of IL-2 secretion from TH1 lymphocytes, as well as even though in a lesser manner that of IL-12 secretion from dendritic cells [12], in association with an inhibition of the secretion of some inflammatory immunosuppressive cytokines, such as IL-6, IL-1 beta and TNF-alpha, and finally an anti-angiogenic effect due to an inhibition of the secretion of both VEGF and hypoxia-inducible factor (HIF), which is the main factor responsible for hypoxia-induced angiogenesis [23]. Then, if we consider that all anticancer therapeutic strategies applied up to now are consisting of an inhibition of cancer cell proliferation, a suppression of tumor-related neoangiogenesis, and a stimulation of the anticancer immunity, MLT would represent the only molecule known up to now able to realize with in itself the overall three major mechanisms of the anticancer cure, represented by antiproliferative, immunostimulating and anti-angiogenic mechanisms of action. Not only, but if we consider that from a synthetic point of view the mechanisms of cancer progression may be substantially identified into six fundamental phases, MLT would represent the only molecule, which has been proven to counteract the overall phases of cancer dissemination [24]. The six fundamental phases of cancer progression may be summarized, as follows: 1) possible and probable pre-existence of a stress-related immuosuppression; 2) malignant transformation of the single cell; 3) alterations of the intercellular junctions, with the following alteration of the intercellular matrix; 4) intercellular matrix alteration-related tumor neoangiogenesis; 5) uncontrolled cancer cell proliferation with the possible direct production of both immunosuppressive cytokines, such as TGF-beta and IL-10 by cancer cells themselves, as well as of VEGF itself; 6) tumor expression of FAS-ligand, which induces the death of $\mathrm{T}$ cells in the case of cell-cell contact with the cancer cell, instead of killing cancer cells through the induction of the apoptotic mechanism. In addition to its direct anticancer action, MLT may also deserve several important palliative effects in cancer cure, namely in the treatment of thrombocytopenia, neoplastic cachexia, anorexia, sleep disturbances and mood depression [25]. Unfortunately, despite these interesting effects, very few clinical studies of MLT in cancer therapy have been performed up to now [26].

\section{Pharmacology of cannabidiol}

At present, CBD has been introduced in cancer cure substantially for only its palliative effects, consisting of antalgic, anxiolytic, antiinflammatory, anticonvulsant and anti-cachectin properties. In contrast, CBD would also to be considered for its potential anticancer effects, due to immunomodulating, antiproliferative, anti-inflammatory and antiangiogenic effects [16-18]. In more detail, CBD is not a cannabinoid agonist, since it would mainly act on vanilloid receptors, and this effects would explain its antalgic effects, but in any case CBD may also exert an anticancer activity, due to both immunomodulating effects, consisting of inhibition of IL-10 secretion and probably also that of IL-17 [16], in association with a stimulation of IL-12 and IL-2 secretions [19], as well as to an inhibition of FAAH activity $[17,18]$, with a consequent increase in the content of the endogenous cannabinoids, which may exert a direct anticancer cytotoxic activity.

\section{Possible clinical neuroendocrine applications in the cure of systemic diseases}

By considering that the main cancer progression-related immune alterations are consisting of diminished functionless of both TH1 cells, with a consequent decline in IL-2 blood levels, and dendritic cells, with a following diminished production of IL-12, in association with a concomitant increased $\mathrm{T}$ reg cell and macrophage system activation, with a consequent enhanced production of IL-10 and TGF-beta by T reg cells, as well as that of IL-6 and TNF-alpha by macrophages [20], at least from a theoretical point of view, cancer-related diminished production of IL-2 and IL-12 and enhanced release of IL-10 and IL-6 may be potentially counteracted by acting of the neuroendocrine regulation of the cytokine network. In more detail, IL-2 deficiency and IL-6 high levels may be treated by MLT [12], while on the other side IL-12 deficiency and IL-10 enhanced secretion may be treated by CBD [16-18]. As far as the autoimmunity is concerned, the autoimmune disease-related enhanced production of IL-17 may be controlled by the cannabinoid agonists, including the endogenous ones, THC, and CBD itself, because of its inhibitory action on FAAH activity, even though it is not a direct cannabinoid agonist. Then, THC would be more effective in the treatment of autoimmune diseases, since autoimmunity-related chronic inflammation would be mainly depend on IL-17 abnormal secretion, which is inhibited by THC [16], whereas CBD could be particularly appropriate in cancer cure by counteracting IL-10dependent suppression of the antitumor immunity and stimulating IL-12-dependent anticancer immunity [19]. The secretion of IL-12 has been proven to be also stimulated by berberine [27]. Then, the immunomodulating properties of CBD could be further enhanced by a concomitant administration of the pineal hormone MLT, because of its ability to stimulate IL-2 secretion from TH1 lymphocytes, or its direct cytotoxic effects against most cancer cell lines. On the same way, the antitumor properties of MLT may be amplified by a concomitant administration of THC itself, which may also exert direct antiproliferative antitumor effects [16-18]. Therefore, the anticancer activity of MLT could be enhanced by both CBD and THC. As far as the activity of MLT in autoimmunity is concerned, more controversial results have been reported in the literature, but most in general they agree with the fact that MLT may improve the clinical status of patients with autoimmune diseases, namely those suffering from bowel inflammatory pathologies [28], because of its anti-inflammatory activity due to inhibition of TNF-alpha and IL-6 secretion, except for the rheumatoid arthritis [12], since either positive or negative effects have been referred. The potential stimulation of autoimmunity-related 

and cannabidiol

inflammation by MLT could be depend on its stimulatory effect on IL-2 release by TH1 lymphocytes [12], with a consequent potential amplification of the inflammatory status, but on the other hand IL-2 may either enhance or inhibit the autoimmune processes, since it may either activate the macrophage system with a following enhanced production of inflammatory cytokines, namely IL-6 itself, or stimulate $\mathrm{T}$ reg system in the presence of high concentrations of TGF-beta [29]. On the same way, IL-12 may either stimulate autoimmunity-related inflammatory response by promoting $\mathrm{TH} 1$ differentiation and a consequent enhanced IL-2 release, or inhibit TH17 functions [20], with a consequent diminished production of IL-17, which has appeared to be one of the most important cytokines in determining the autoimmune reactions [30]. Finally, the immunosuppressive effects of mu-opioid agonists, including morphine, consisting of inhibition of IL-2 and stimulation of IL-10 secretion [8-10], could be potentially therapeutic in the autoimmune diseases, but the potential stimulatory effect of muopioid agonists on IL-17 secretion could verify the benefit due to their inhibition on IL-2 and stimulation on IL-10 [31].

\section{Clinical results with MLT and CBD in human systemic diseases}

At present, it becomes to be possible to interpret are cancer cure as a simple reestablishment of the functionless of cytokine network of the status of health, consisting of a perfect equilibrium between immunostimulatory and immunosuppressive cytokines, since a prevalence of immunostimulatory cytokines may allow to autoimmune pathologies, whereas an exaggerated production of immunosuppressive cytokines may predispose to cancer development. Therefore, because of the low clinical availability of IL-2 and the complete lack of IL-12 availability for clinical studies, from a practice clinical point of view similar results, consisting of an enhanced endogenous secretion of both IL-2 and IL-12, could be potentially reached by a neuroendocrine approach elaborated on the basis of the knowledge of the physiological neuroendocrine control of the cytokine network, such as that represented by the concomitant administration of MLT and CBD, which could constitute a simple, nontoxic and less expensive therapy of cancer, at least for those advanced cancer patients, for whom no other standard therapy may be available, by reserving the classical cancer immunotherapy with cytokines, such as IL-2 and IL-12, for the only patients, for whom the association between MLT and CBD is not sufficient to increase TH1 lymphocyte and mature dendritic cell number, and the consequent increase in IL-2 and IL-12 blood concentrations, respectively. Unfortunately, despite the great number of experimental studies confirming the immunomodulating properties of some neurohormones or neuroactive molecules, namely MLT, $\mathrm{CBD}, \mathrm{THC}$, and mu-opioid antagonists, very few clinical studies have been performed up to now to evaluate the real effects of their possible combinations as a neuroimmune approach in the cure of systemic diseases, including cancer and autoimmune diseases, in an attempt to correct cancer and autoimmunity-related alterations in cytokine secretions by acting on the neuroendocrine regulation of cytokine network itself, instead of a direct artificial action on the immune effects of cytokines, as in the immunotherapy with monoclonal antibodies. In patients with disseminated cancer and eligible for the only palliative cure, high-dose MLT has appeared to prolong the survival time through a stimulation of the antitumor immunity by activating $\mathrm{T}$ lymphocyte and inhibiting macrophage systems [32], and MLT effects would seen to be further enhanced by a concomitant administration of both THC and CBD [33]. Therefore, randomized studies will be needed to establish whether the immunomodulating properties of MLT may be better amplified by CBD either in cancer therapy or in the treatment of autoimmunity.

\section{Conclusion}

Until we will consider the clinical application of neuroactive immunomodulating molecules, such as MLT and CBD, as a simple pallative therapy of the untreatable human systemic diseases, no further advances in the Clinical Practices may be reached. On the contrary, by taking into consideration their effects on cell proliferation and immune functionless, it could be possible to control the clinical course of most human systemic diseases by acting on the central neuroendocrine regulation of the immune system through a modulation of the cytokine network, by abrogating the non-scientific difference between curative and palliative therapy of cancer and autoimmunity.

\section{References}

1. Grivennikov SI, Greten FR, Karin M (2010) Immunity inflammation and cancer. Cell 295: 883-899. [Crossref]

2. Dong C (2008) Regulation and pro-inflammatory function of interleukin-17 family cytokines. Immunol Rev 226: 80-86. [Crossref]

3. Abdel Galli SM, Ezzeldin N, El-Boshy ME (2015) The role of serum IL-17 and IL-6 as biomarkers of disease activity and prediction of remission in patients with lupus nephritis. Cytokine 76: 280-287. [Crossref]

4. Zou W (2006) Regulatory T cells, tumor immunity and immunotherapy. Nat Rev Immunol 6: 295-307. [Crossref]

5. Nahrendorf M, Swirski FK (2016) Abandoning M1/M2 for a network model of macrophage function. Circulation Res 119: 414-417. [Crossref]

6. Reiss M (1999) TGF-beta and cancer. Microb Infect 1: 1327-1347. [Crossref]

7. Paramalingam SP, Thumboo J, Vasoo S, Thio ST, Tse C, et al. (2007) In vivo pro- and anti-inflammatory cytokines in normal and patients with rheumatoid arthritis. Ann Acad Med Singapore 36: 96-99. [Crossref]

8. Manfredi B, Sacerdote P, Bianchi M (1993) Evidence for an opioid inhibitory tone on T cell proliferation. J Neuroimmunol 44: 43-46. [Crossref]

9. Ondicova C, Mravec B (2010) Role of nervous system in cancer aetiopathogenesis. Lancet Oncol 11: 596-601. [Crossref]

10. Cioca DP, Watanabe N, Isobe M (2000) Apoptosis of peripheral blood lymphocytes is induced by catecholamines. Jpn Heart J 41: 385-398. [Crossref]

11. Lissoni P, Resentini M, Mauri R, Esposti D, Esposti G, et al. (1986) Effects of tetrahydrocannabinol on melatonin secretion in man. Horm Metabol Res 18: 77-78. [Crossref]

12. Dewey WL (1986) Cannabinoid pharmacology. Pharmacol Rev 38: 151-178. [Crossref]

13. Russo EB (2004) Clinical endocannabinoid deficiency (CECD). Neuro Endocrinol Lett 25: $31-39$.

14. Brzezinski A (1997) Melatonin in humans. N Engl J Med 336: 185-195. [Crossref]

15. Grotenhermen F (2004) Pharmacology of cannabinoids. Neuro Endocrinol Lett 25: 1423. [Crossref]

16. Smith SR, Terminelli C, Denhartdt G (2000) Effects of cannabinoid receptor agonist and antagonist ligands on production of inflammatory cytokines and anti-inflammatory intereukin-10 in endotoxemic mice. J Pharmacol Exp Ther 293: 136-150. [Crossref]

17. Nagarkatti P, Pandey R, Rieder SA, Hedge VL, Nagarkatti M, et al. (2009) Cannabinoids as novel anti-inflammatory drugs. Future Med Chem 1: 1333-1349. [Crossref]

18. Lissoni P, Messina G, Porro G, Trampetti R, Lissoni A, et al. (2018) The modulation of the endocannabinoid system in the treatment of cancer and other systemic human diseases. Glob Drugs Therap 3: 1-4.

19. Sacerdote P, Martucci P, Vaccani A, Bariselli F, Panerai AF, et al (2005) The non psychoactive component of marijuana modulates chemotaxis and IL-10 and IL-12 production of murine macrophages both in vivo and in vitro. $J$ Neuroimmunol 159: 97-105. [Crossref]

20. Lissoni P (2017) Therapy implications of the role of interleukin-2 in cancer. Exp Rev Clin Immunol 13: 491-498. [Crossref] 
Lissoni P (2019) A Review on the neuroendocrine regulation of cytokine secretion: Possible modulation of the cytokine network by the pineal hormone melatonin and cannabidiol

21. Bartsch C, Bartsch H (1999) Melatonin in cancer patients and in tumor-bearing animals. Adv Exp Med Biol 467: 247-264. [Crossref]

22. Conti A, Maestroni GJM (1995) The clinical immunotherapeutic role of melatonin in Oncology. J Pineal Res 19: 103-110. [Crossref]

23. Park SY, Jang WJ, Yi EY, Jung Y, Jeong JW, et al. (2010) Melantonin suppresses tumor angiogensis by inhibiting HIF-1 alpha stabilization under hypoxia. $J$ Pineal Res 48 : 178-184. [Crossref]

24. Reiter RJ (2004) Mechanisms of cancer inhibition by melatonin. J Pineal Res 37: 213 214. [Crossref]

25. Lissoni $\mathrm{P}$ (2000) Is there a role for melatonin in supportive care?. Supp Care Cancer 10: 110-116. [Crossref]

26. Millis E, Wu P, Seely D, Guyatt G (2005) Melatonin in the treatment of cancer: a systematic review of randomized controlled trials and meta-analysis. J Pineal Res 39 : 360-366. [Crossref]

27. Kim TS, Kang BY, Cho D, Kim SH (2003) Induction of IL-12 production by berberine, a benzodioxoloquinolizine alkaloid derivate. Immunology 109: 407-414. [Crossref]
28. Lin GJ, Huang SH, Chen SJ, Wang CH, Chang DM, et al. (2013) Modulation by melatonin of the pathogenesis of inflammatory autoimmune diseases. Int J Mol Sci 14 11742-11766. [Crossref]

29. He J, Zhang X, Wei Y, Sun X, Chen Y, et al. (2016) Low-dose interleukin-2 treatment selectively modulates CD4+ T cell subsets in patients with systemic lupus erythematosus. Nat Med 22: 991-993. [Crossref]

30. Chen Z, Tato CM, Muul L, Laurence A, O'Shea JJ, et al. (2007) Distinct regulation of IL-17 in human helper T lymphocytes. Arthritis Rheum 56: 2936-2946. [Crossref]

31. Lissoni P, Messina G, Cenaj V, Rovelli F, Porro A, et al. (2018) The role of IL-17 secretion in mediating the influences of stress on cancer and other human systemic diseases. MOJ Lymphol Phebol 2: 31-34.

32. Lissoni P, Rovelli F, Brivio F, Messina G, Lissoni A, et al. (2018) Five year-survival with high-dose melatonin and other antitumor pineal hormones in advanced cancer patients eligible for the only palliative therapy. Res J Oncol 2: 1-7.

33. Lissoni P, Messina G, Porro G, porta E, Nosetto L, et al. (2016) A psychoneuroendocrino-immune (PNEI) approach to enhance the efficacy of radiochemotherapy in glioblastoma. J J Rad Oncol 3: 29-32.

Copyright: (2019 Lissoni P. This is an open-access article distributed under the terms of the Creative Commons Attribution License, which permits unrestricted use, distribution, and reproduction in any medium, provided the original author and source are credited. 